\title{
Application of dual-genome oligonucleotide array-based comparative genomic hybridization to the molecular diagnosis of mitochondrial DNA deletion and depletion syndromes
}

\author{
A. Craig Chinault, PhD, Chad A. Shaw, PhD, Ellen K. Brundage, BA, Lin-Ya Tang, MS, \\ and Lee-Jun C. Wong, PhD
}

\begin{abstract}
$\sqrt{\overline{\overline{\sqrt{x}}}}$ se: Mitochondrial disorders constitute a group of clinically and genetically heterogeneous diseases for which molecular diagnosis has been a challenge. The current procedures for diagnosis of mitochondrial DNA deletion and depletion syndromes based on Southern analysis and quantitative polymerase chain reaction are particularly inefficient for determining important parameters of deletion endpoints and percent heteroplasmy. We have developed an improved approach for routine analyses of these disorders in a clinical laboratory. Methods: A customdesigned oligonucleotide array-based comparative genomic hybridization platform was developed to provide both tiled coverage of the entire 16.6-kb mitochondrial genome and high-density coverage of nuclear genes involved in mitochondrial biogenesis and function, for quick evaluation of mitochondrial DNA deletion and depletion. Results: For initial validation, the performance of this array was characterized in 20 samples with known mitochondrial DNA deletions and 12 with apparent depletions. All previously known deletions were clearly detected and the break points were correctly identified by the oligonucleotide array-based comparative genomic hybridization, within the limits of resolution of the array. The extent of mitochondrial DNA depletion and the percentage of deletion heteroplasmy were estimated using an automated computational approach that gave results comparable to previous methods. Conclusions from subsequent application of this approach with $>300$ new clinical samples have been in $100 \%$ concordance with those from standard methods. Finally, for one sample, we were able to identify an intragenic deletion in a nuclear gene that was responsible for the observed mitochondrial DNA depletion. Conclusion: We conclude that this custom array is capable of reliably detecting mitochondrial DNA deletion with elucidation of the deletion break points and the percentage of heteroplasmy. In addition, simultaneous detection of the copy number changes in both nuclear and mitochondrial genomes makes this dual genome array of tremendous value in the diagnoses of mitochondrial DNA depletion syndromes. Genet Med 2009:11(7): 518-526.
\end{abstract}

Key Words: aCGH diagnosis of mitochondrial disorders, mitochondrial DNA deletion, mtDNA depletion, oligonucleotide aCGH, dual genome disorder

From the Department of Molecular and Human Genetics, Baylor College of Medicine, Houston, Texas.

Lee-Jun C. Wong, PhD, Department of Molecular and Human Genetics, Baylor College of Medicine, One Baylor Plaza, NAB 2015, Houston, TX 77030. E-mail: ljwong@bcm.edu.

Disclosure: The authors declare no conflict of interest

Submitted for publication January 26, 2009.

Accepted for publication April 7, 2009.

Published online ahead of print June 19, 2009.

DOI: $10.1097 /$ GIM.0b013e3181abd83c
$\mathrm{M}$ itochondrial disorders can be caused by mutations in both nuclear and mitochondrial genomes. In the past, diagnosis of such disorders has been a great challenge due to the extreme degree of clinical and genetic heterogeneity and the complex interplay between dual genomes involved in the biogenesis and maintenance of the mitochondrial genome, as well as the respiratory and metabolic functions in which mitochondria play a crucial role. Mitochondrial DNA (mtDNA) deletion is a common cause of disorders such as Kearns Sayre, Pearson, myopathy, maternally inherited diabetes/hearing loss, chronic progressive external ophthalmoplegia, and multisystemic diseases. In contrast, mtDNA depletion syndrome is caused by mutations in nuclear genes that are responsible for the biosynthesis of mtDNA such as DNA polymerase gamma (POLG), or the maintenance of deoxynucleotide pools such as deoxyguanosine kinase $(D G U O K)$ and thymidine kinase 2 (TK2). The goal of this work was to develop an approach for simultaneous detection of copy number changes affecting either nuclear or mitochondrial genes to complement DNA sequencing analyses.

Comparative genomic hybridization $(\mathrm{CGH})$ on microarrays constructed with DNA from bacterial artificial chromosome (BAC) clones $^{1-3}$ or, more recently, synthetic oligonucleotides ${ }^{4,5}$ as probes has been widely used in recent years as a highly multiplexed screen for large chromosomal deletions and duplications at the whole genome level. Alternatively, high-density coverage of a specific gene or group of genes of interest has been used to increase the sensitivity for detection of intragenic copy number changes. $6,7 \mathrm{We}$ have demonstrated that such an oligonucleotide array is of great utility to screen for mutations in nuclear genes involved in metabolic and mitochondrial disorders when used in conjunction with direct DNA sequencing, particularly in autosomal recessive diseases when only one heterozygous mutant allele is detected. ${ }^{7}$

To date, the application of oligonucleotide array-based CGH $(\mathrm{aCGH})$ for detection of copy number changes has been focused on nuclear chromosomes without the inclusion of the mitochondrial genome. Although this genome constitutes an extremely small portion of the genetic information in a cell, disruption of either its primary DNA structure or reduction in its normal cellular copy number can lead to devastating disorders. ${ }^{8-11}$ Because molecular defects in numerous nuclear genes can severely affect the mtDNA integrity and copy number, ${ }^{12-22}$ it would be of great utility to have available an oligonucleotide array for the entire $16.6 \mathrm{~kb}$ mitochondrial genome in conjunction with a group of nuclear genes that are known to cause mitochondrial disorders for the evaluation of mitochondrial disease. In this report, we describe the application of such a custom-designed $44 \mathrm{~K}$ array for the purpose of molecular diagnosis of mitochondrial disorders. We demonstrate the power of this approach for rapid determination of mitochondrial genome deletion and depletion by comparison with methods that are in 
routine use. In addition, we show how a nuclear gene deletion affecting mitochondrial genome copy numbers can be analyzed simultaneously by array-CGH.

\section{MATERIALS AND METHODS}

\section{Detection of mtDNA deletion}

Total DNA was extracted from peripheral blood leukocytes, skin fibroblasts, liver, or muscle tissue using commercially available DNA isolation kits (Gentra Systems Inc., Minneapolis, MN) according to the manufacturer's protocols. All DNA samples with mtDNA deletions were originally detected by Southern blot analysis as described previously 23,24 and in the legend of Figure 1. The deletion mutant heteroplasmy was estimated from the Southern blot by densitometric scanning.

\section{Determination of deletion break points by polymerase chain reaction}

Deletion break points were determined by polymerase chain reaction $(\mathrm{PCR})$ amplification using primers encompassing the maximal deletion region followed by sequencing of the junction fragment using the BigDye Terminator Cycle Sequencing kit (version 3.1) and analyzed on an ABI3730XL automated DNA sequencer with Sequencing Analysis Software v5.1.1 (Applied Biosystems, Foster City, CA). DNA sequences were analyzed using Mutation Surveyor version 2.6.1 and the GenBank sequence AC_000021 was used as the reference sequence for mitochondrial whole genome.

\section{Real-time quantitative PCR analysis of mtDNA copy number}

mtDNA copy number was evaluated by real-time quantitative PCR (qPCR) as previously described. ${ }^{25}$ MtDNA and nuclear DNA copy numbers were determined by real-time qPCR using specific primers for the mitochondrial tRNA ${ }^{\text {Leu(UUR) }}$ gene region (mtF3212-3231 and $m t R 3319-3300)$ and the nuclear gene $\beta$-2-microglobulin $(\beta 2 M)$ using the specific primers, $\mathrm{ntF}$ 589-613 and ntR 674-653 in the 3'UTR (GI:37704390). ${ }^{25}$ All samples were assayed in duplicate and confirmed by a repeat analysis in a second run. Each $10 \mu \mathrm{L}$ PCR reaction contained

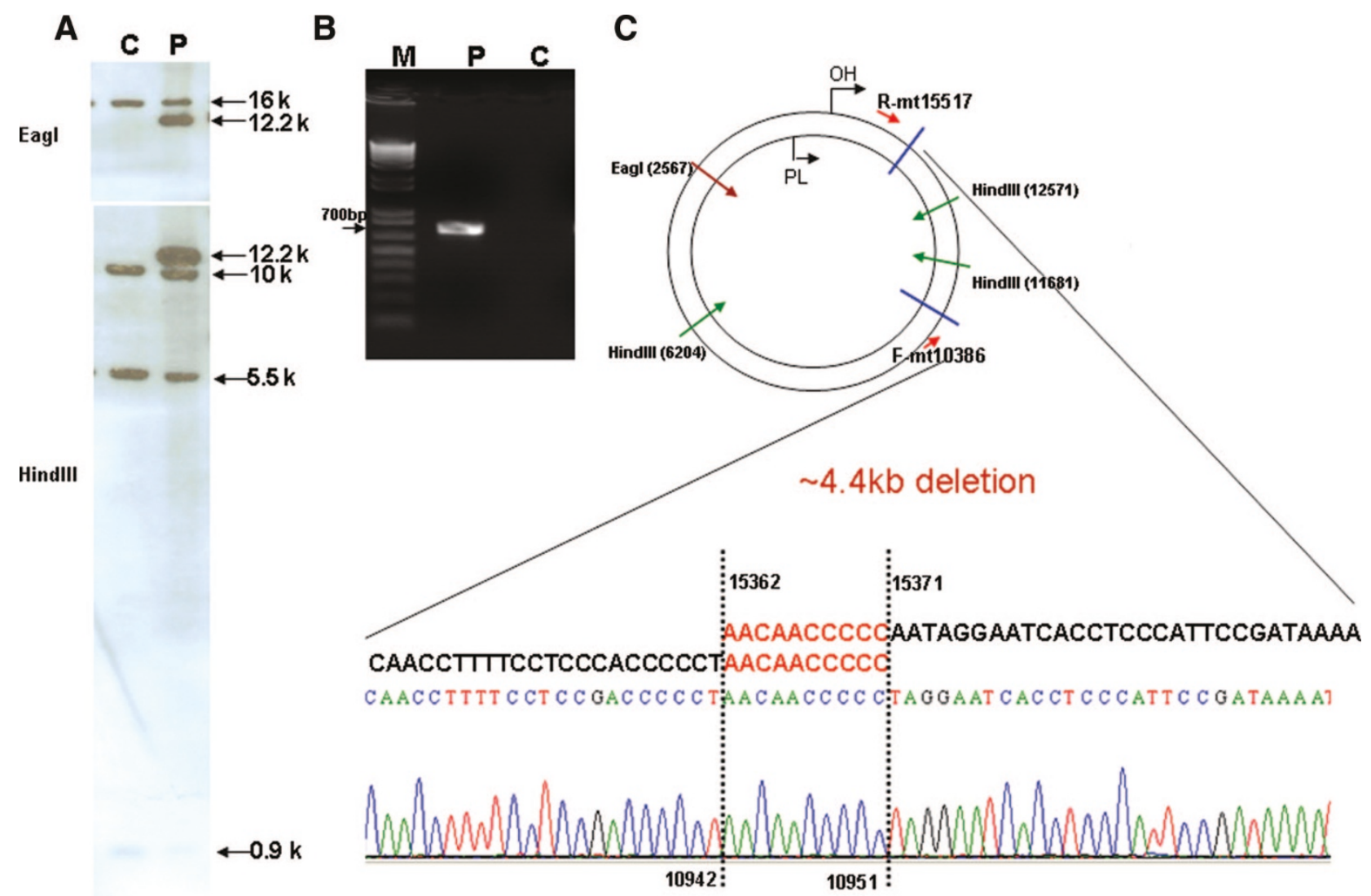

Fig. 1. Characterization of mtDNA deletion. A, Southern blot: DNA samples from the patient (P) and normal control (C) were digested with Eagl (upper panel) and Hind III (bottom panel). MtDNA was detected by Southern blot. There are 1 Eag I cutting site and 3 Hind III cutting sites in the wild-type mtDNA. Eagl linearizes the DNA to give a full length of 16.6 $\mathrm{kb}$ fragment. The smaller band $(12.2 \mathrm{~kb})$ indicates the deletion mtDNA. The coexistence of $16.6 \mathrm{~kb}$ and $12.2 \mathrm{~kb}$ bands indicates that the deletion mtDNA is in a heteroplasmic state. HindIIl digestion gave three bands, 10.2, 5.5, and 0.9 kb. The $12.2 \mathrm{~kb}$ band in the P lane indicates the heteroplasmic deletion mtDNA molecule. B, PCR: The primers F-mt10386 and R-mt15517 amplified a DNA fragment of 702 bp in patient's DNA sample. The PCR condition failed to amplify the undeleted wild-type mtDNA in control sample because the primers are too far apart. C, Primer positions and deletion junction sequence. Note the $10 \mathrm{bp}$ direct repeat at the junction. 
5X iTaqSYBR Green Supermix with ROX (Bio-Rad, Hercules, CA), $300 \mathrm{nmol} / \mathrm{L}$ of each primer, and $0.3 \mathrm{ng}$ of total DNA. The real-time PCR conditions were 2 minutes at $50^{\circ} \mathrm{C}$ and 10 minutes at $95^{\circ} \mathrm{C}$, followed by 45 cycles of 15 seconds of denaturation at $95^{\circ} \mathrm{C}$ and 30 seconds of annealing/extension at $62^{\circ} \mathrm{C}$. The fluorescent signal intensity of PCR products was recorded and analyzed on a 7900HT fast real-time PCR system (Applied Biosystems, Foster City, CA) using SDS v2.2.2 software. The total amount of mtDNA (referred to as the relative mtDNA copy number) in DNA samples from patients and control subjects were calculated from the difference in threshold cycle numbers of nuclear gene and mtDNA, $\Delta \mathrm{Ct}$ value, where the mean amount of mtDNA/cell $=2(2-\Delta \mathrm{Ct})$, accounting for the two copies of $\beta 2 M$ in each cell nucleus.

\section{Array-CGH}

A custom array was made using the Agilent oligonucleotide $4 \times 44 \mathrm{~K}$ microarray platform (Agilent Technologies, Santa Clara, CA). This array had coverage for approximately 150 nuclear genes that are related to metabolic pathways and mitochondrial biogenesis and function at an average probe spacing of about 250 to $300 \mathrm{bp}$ per oligonucleotide probe. The names of the genes and disorders, as well as the chromosomal locations and OMIM numbers are listed on our website (www.bcmgeneticlabs. org). The most recent versions of this array also include lowresolution backbone coverage. In addition, 6400 oligonucleotide probes covering the $16.6 \mathrm{~kb}$ of the entire mitochondrial genome in both forward and reverse directions were also included. The 37 genes encoded by the mitochondrial genome and their nucleotide positions can be found in http://www.mitomap.org. This array is referred to as a MitoMet array.

For each aCGH experiment, $1 \mu \mathrm{g}$ each of purified patient DNA and gender-matched reference DNA was digested with AluI (10 units) and RsaI (10 units) (Promega, Madison, WI) and differentially labeled by random priming with a Bioprime $\mathrm{CGH}$ Labeling Module (Invitrogen, Carlsbad, CA) with cyanine-5 and cyanine-3 fluorescent dyes (Perkin-Elmer, Waltham, MA), respectively. Hybridization was carried out for at least 20 hours at $65^{\circ} \mathrm{C}$ in a rotating microarray hybridization chamber and then washed according to the manufacturer's protocols (Agilent Technologies, Santa Clara, CA). The slide hybridization results were scanned into image files using a GenePix Model microarray 4000B scanner (Molecular Devices, Sunnyvale, CA) at high and low photomultiplier tube (PMT) settings or an Agilent microarray scanner using the extended dynamic range function to prevent signal saturation for mitochondrial probes. The array features were then located and quantified using the Agilent Feature Extraction v9.1 software and text file outputs were analyzed for relative copy number changes using the Agilent $\mathrm{CGH}$ Analytics software program with a modified design file that allows visualization of mitochondrial data, as well as the analysis programs developed in-house as described later.

\section{Segmentation and heteroplasmy analysis}

Although visual inspection and simple calculations on relative copy number allowed quite accurate interpretation of the data, we also developed an objective statistical approach to data analysis for automated high-throughput purposes. In this analysis, poor performing oligonucleotides were identified as those oligonucleotides where the opposite strand features showed high variance (median standard deviation across cases $>0.15$ on the log scale) or where the data were unusually highly spread across cases (interquartile range, across cases $>0.85$ ). The identified data points were observed to be more or less uniformly spread across the mitochondrial genome and to be un- correlated with genomic GC content. Subsequent to the filtering step, circular binary segmentation was performed on the logratio intensity data for the mitochondrial genome within each patient. Because the raw log-ratio data were observed to be relatively noisy, an additional median smooth calculation was performed to remove additional technical noise by taking the middle value from every three using the smooth method in R. The copy number segments determined by the segmentation algorithm were then determined using circular binary segmentation as implemented in the algorithm by Olshen et al. ${ }^{26}$ The resulting segments were then subjected to postprocessing to merge all segments within each patient when (a) disjoint segments were within 500 bp of each other, (b) had the same directionality (i.e., loss or gain), and (c) where the difference in magnitudes between segments was $<0.75$ on the log scale. The subsequent intervals were used for both the calculation of heteroplasmy and for comparison with the deletion coordinates from the PCR/sequencing method.

The degree of deletion heteroplasmy from the oligonucleotide aCGH data were determined by using an in-house computational method that adopted a mixture model approach. Under this method, the difference in the median normalized log-ratio between the deleted segment and the nondeleted segment was exponentiated and one minus this value was taken as an estimate of heteroplasmy. This calculation is consistent with the biological model that the population of mitochondria consists of a mixture of two genotypes, one without the deletion event and one with. In this case, the heteroplasmy calculation estimates the percent deleted.

\section{RESULTS}

Current diagnostic analysis of mtDNA deletions involves Southern blotting and restriction-fragment-length polymorphism analysis to detect and localize a deletion followed by PCR amplification and sequencing of junction fragments to determine the precise break points. ${ }^{23,24}$ The quantification of deletion mutant heteroplasmy is based on densitometric scanning or phosphorimaging of the signal intensities of linearized deletion and nondeletion DNA molecules. As shown in Figure 1A (upper panel), heteroplasmic deletion can be detected by linearization of the circular mtDNA with a single cut restriction enzyme, such as EagI; the $16.6 \mathrm{~kb}$ band represents the intact linearized mtDNA whereas the faster moving smaller band represents the deletion mtDNA molecule. The deletion mutant heteroplasmy can be estimated by densitometric scanning of these two bands. However, if the deletion is small and the two bands are not well separated, it is difficult to estimate the percent of heteroplasmy. Thus, Southern analysis using another restriction enzyme, such as HindIII, that cuts the mtDNA molecule into three smaller fragments as demonstrated in Figure 1A (lower panel) may be necessary for the estimation of percentage of heteroplasmy. ${ }^{23,24}$ To determine the deletion break points, PCR primers encompassing the deletion region are used to amplify the junction fragment, which is then sequenced (Fig. 1, B-D). These sequential procedures are time consuming and very often involve radioactive material. Furthermore, this method does not detect overall mtDNA copy number changes; for this, a real-time qPCR analysis must be performed using probes specific for nuclear and mitochondrial genes. ${ }^{25}$

By comparison, when DNA samples with mitochondrial deletions were analyzed on the custom MitoMet array, the affected regions were revealed immediately and the percentage of deletion heteroplasmy could be easily estimated from the same data. Figure 2 shows four representative examples of DNA samples 


\begin{tabular}{|c|c|c|c|c|c|c|c|c|c|c|c|c|c|c|c|}
\hline tRNA & $\mathrm{F}$ & v & L & IQM & WANCY & SD & K & & & G & R & HSL & & E & $\mathrm{TP} \longrightarrow \mathrm{C}$ \\
\hline rRNA & 125 & $16 \mathrm{~S}$ & ND1 & ND2 & $\mathrm{COI}$ & |coll & ATP8 & ATP6 & collI & ND3 & ND4 & ND5 & ND6 & CYTB & Dloop \\
\hline
\end{tabular}

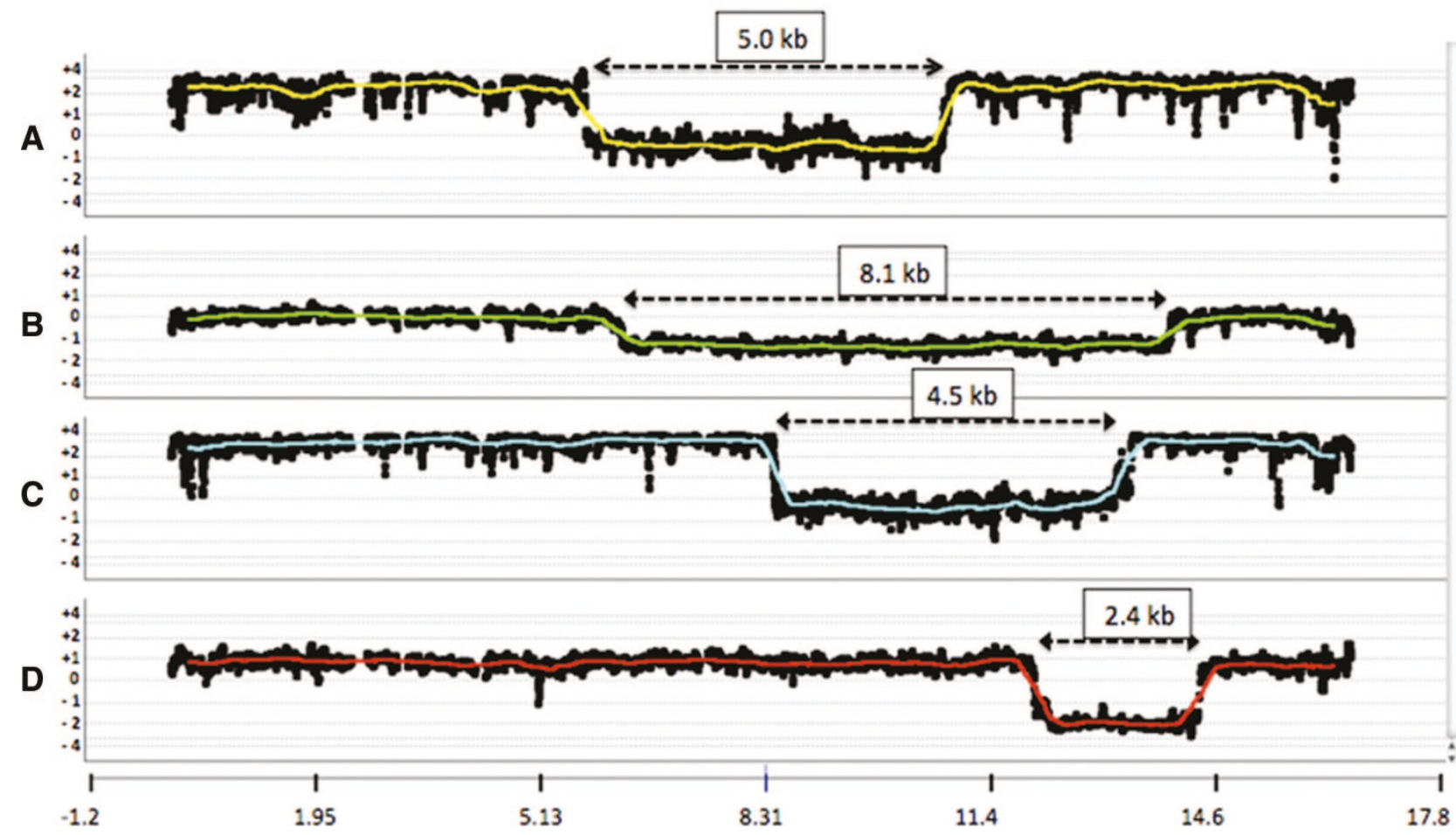

Fig. 2. Four representative array $\mathrm{CGH}$ analyses showing mitochondrial genome deletions. The results are plotted on a log2 scale (vertical axis) as a function of oligonucleotide position in the mitochondrial genome position (horizontal axis). DNA samples were from muscle, blood, muscle, and skin for panels A, B, C, and D, respectively, using a blood DNA sample from a 65 -year-old individual as the reference. The $y$-axis is showing that the mtDNA copy number in muscle and skin is about 10 and 2 times higher than that in blood. Both the extent of deletions and the relative populations of intact and deleted mitochondrial genomes (\% heteroplasmy) are variable. The 37 genes encoded by the mitochondrial genome are depicted on the top. Zero designates the origin of replication.

from patients with mitochondrial genome deletions of various sizes, positions, and percentages of deletion mutant heteroplasmy. These four DNA samples were from muscle, blood, muscle, and skin for panels $\mathrm{A}, \mathrm{B}, \mathrm{C}$, and $\mathrm{D}$, respectively, using a blood DNA sample from a 60-year-old individual as the reference. It is obvious that the mtDNA copy number varies among different tissues.

With the high density of overlapping oligonucleotides covering both strands of the mtDNA at an average spacing for start positions of $\sim 3$ nucleotides, the approximate size and endpoints of the deletions could be determined readily from the known sequence coordinates of the overlapping probes on the array. The MitoMet aCGH was applied to analyze 20 samples that previously tested positive by the traditional Southern blot analysis. Results from both the classical approaches and the array analyses for all these cases are summarized in Table 1. It is clear that results, including the deletion break points, the size of deletion, and the percentage of deletion heteroplasmy, obtained from the aCGH are consistent with those determined by the traditional Southern blotting and sequencing analysis.

DNA depletion or proliferation analyses from microarray data are less straightforward because mitochondrial copy number is known to differ in a tissue-specific pattern. When muscle DNA is used as the control for a muscle specimen, a copy number ratio of 1:1 is expected, thus, giving a $\log 2$ value of 0 . Although the exact quantification of mitochondrial copy number is difficult, significant changes from normal levels can still be recognized. This is demonstrated in Table 2, which summarizes the results of $\mathrm{qPCR}$ and aCGH analyses using different reference DNA samples. Those in Group 1 were the typical age and tissue-matched controls used for qPCR. Control 2 used for qPCR is the same reference DNA that was used for aCGH. As revealed by Case 32, for example, when the control samples were age- and tissue-type matched, the mtDNA content measured by qPCR and aCGH was consistent (5 and 7\%). In Case 37 , mtDNA depletion in liver (12\% of control liver sample) was clearly detected when age- and tissue-type matched control was used. However, when blood was used as control, values of $316 \%$ and $261 \%$ were obtained by qPCR or aCGH, respectively. This high mtDNA content reflects the combination of the different abundance of mitochondria in liver compared with blood (approximately 10-fold higher in muscle and liver), the depletion in the patient's liver sample (about 12\%), and the age difference (approximately 2-fold). Case 39 is the same patient as Case 38, except that the tissue analyzed is different. It is interesting to note that in the patient with the myopathic form of mtDNA depletion syndrome, severe reduction in mtDNA content was observed in both muscle and liver by qPCR and aCGH 


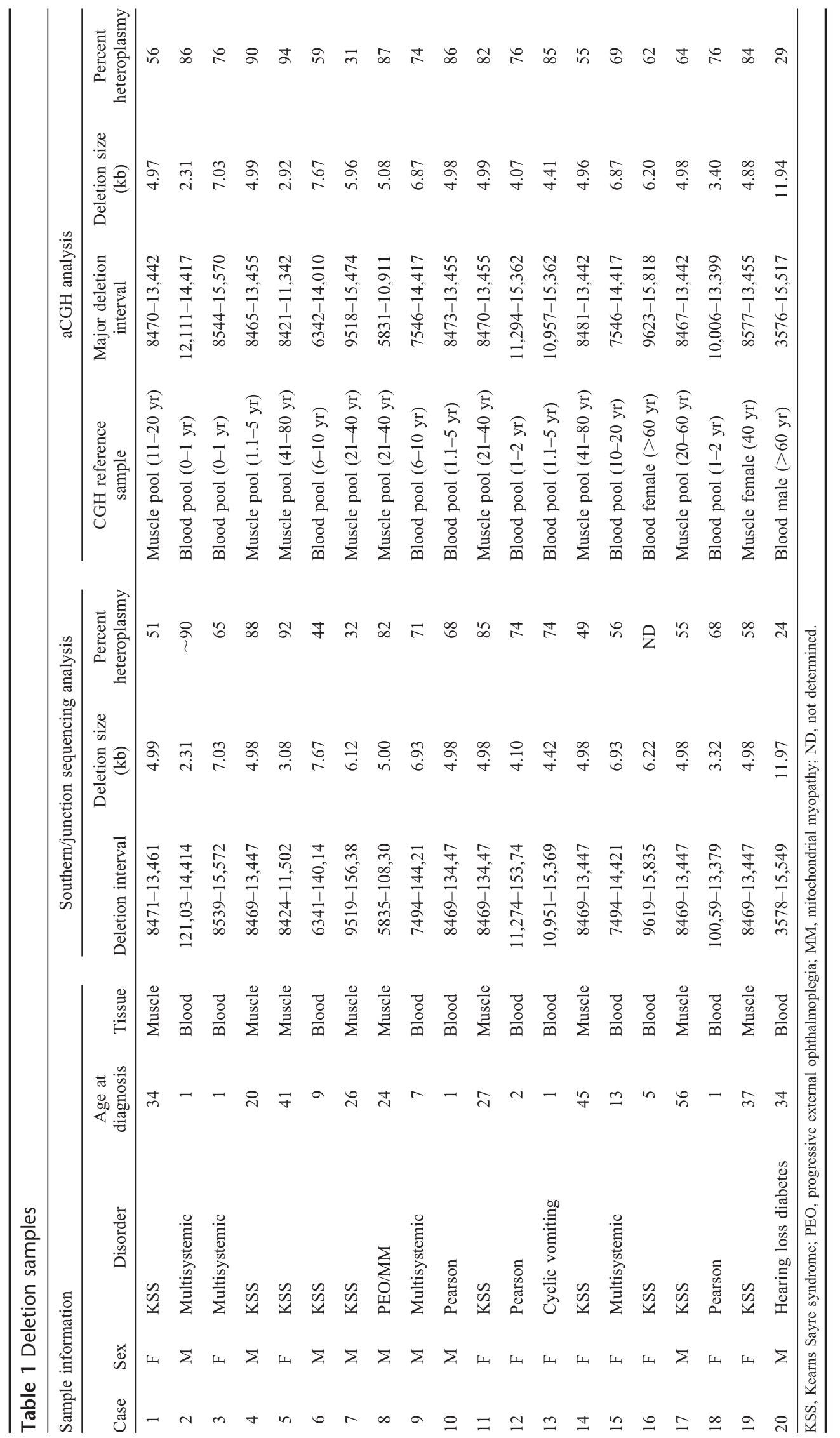




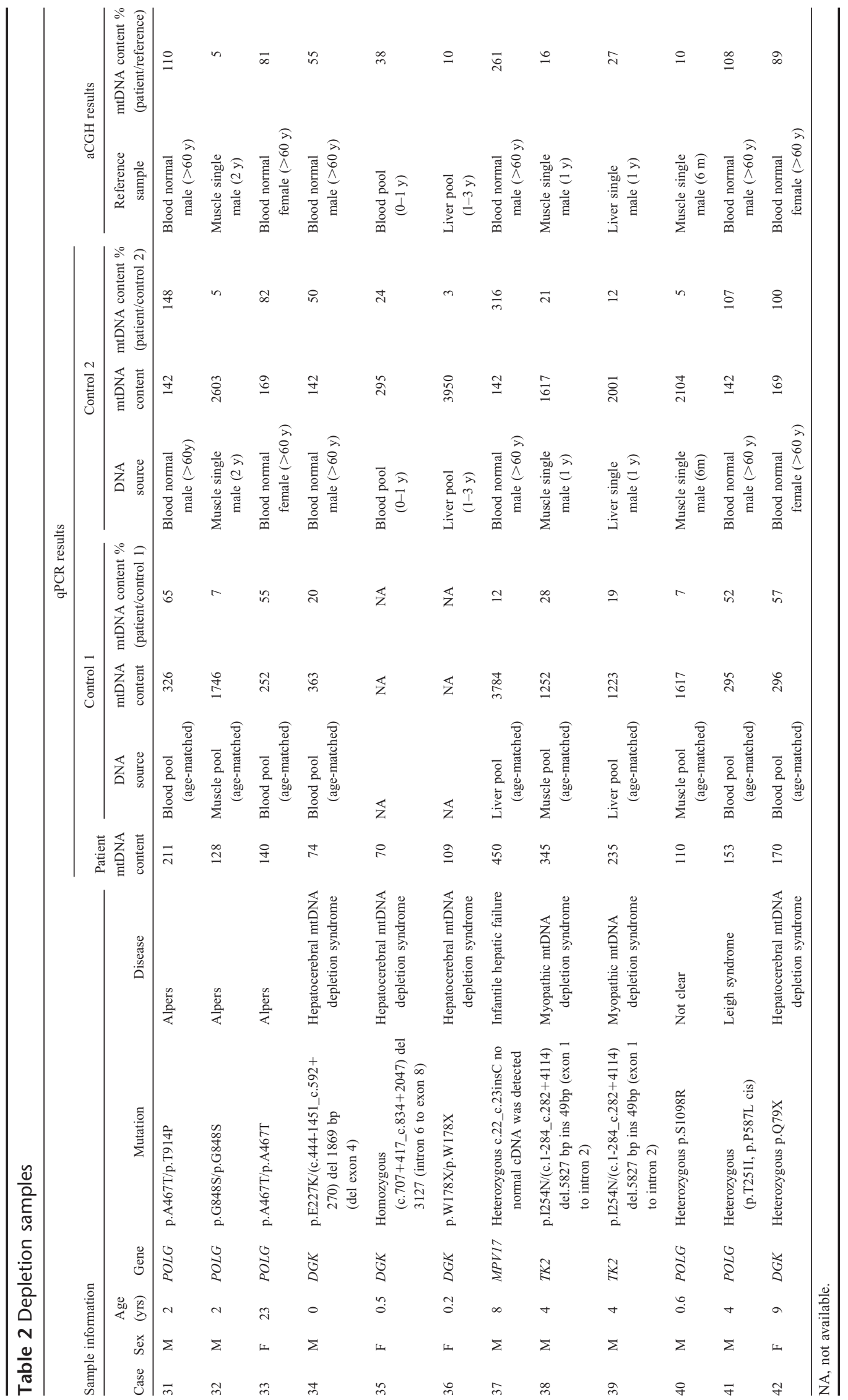




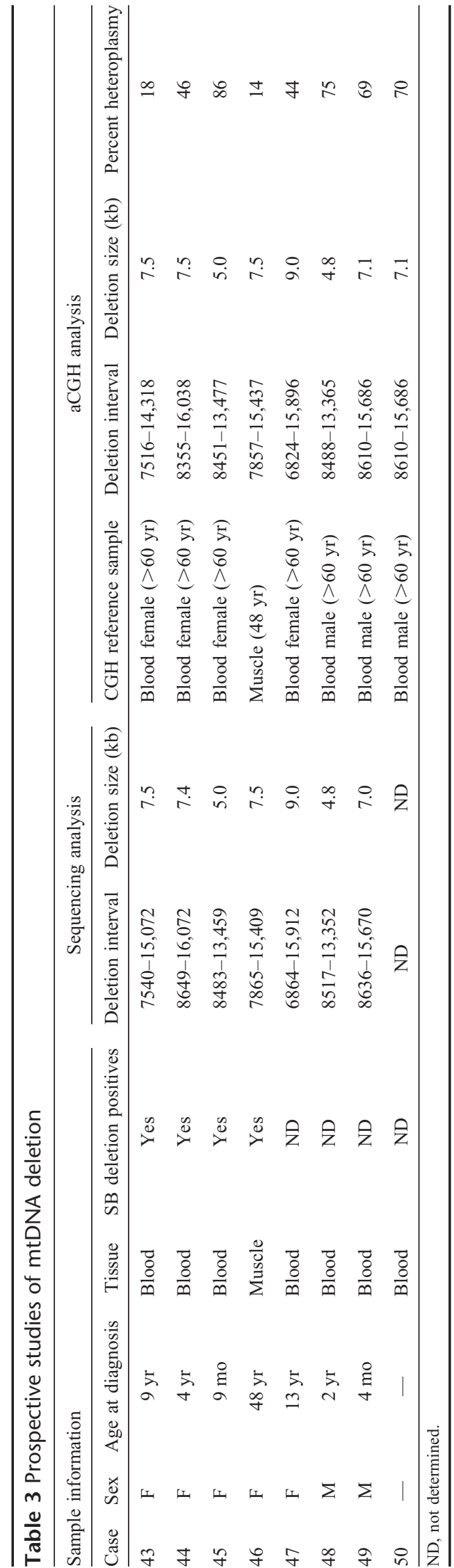

analyses using matched controls. In Case 33, reduction in mtDNA content was detected by qPCR when age-matched blood sample was used as control. The reduction in mtDNA content was not readily detected when a non-age-matched blood sample was used for control either in qPCR or aCGH analysis. It is apparent from the data in Table 2 that selection of the proper reference for aCGH is crucial in many cases to identify cases of mitochondrial depletion. It is clear that some patients with depletion in muscle by qPCR failed to show abnormal copy number in blood compared with reference DNA from blood (e.g., cases 31, 41, and 42).

Table 3 summarizes the results from analysis of 326 new patients using this array approach. Various heteroplasmic mtDNA deletions were detected in eight DNA samples; the estimated break points, size of deletion, and percentage of deletion heteroplasmy detected by aCGH are listed. The exact break points of each deletion were subsequently confirmed by PCR/sequencing (Table 3). Case 50 was a blinded sample of Case 49 analyzed for internal proficiency testing purposes. The aCGH pattern was identical to Case 49 and the same break points, size of deletion, and percentage of deletion heteroplasmy were identified.

Finally, as an example of the complementary power of this approach in correlating nuclear gene mutation with observed abnormal mitochondrial copy, we studied the $T K 2$ gene in detail in one family. When sequencing was performed on the TK2 gene of the parents of a deceased patient diagnosed with myopathic form of mtDNA depletion syndrome, a novel heterozygous point mutation, p.I254N, was identified in the father. No mutant allele was detected in the mother's gene. Because TK2 deficiency is an autosomal recessive disorder, we speculated that the second mutant allele could be an intragenic deletion of the TK2 gene. Therefore, the DNA sample from the mother's blood was analyzed with the MitoMet oligonucleotide aCGH.

As shown in Figure 3A, significant depletion of the mitochondrial copy number was seen in both liver and muscle when aCGH was performed with matched-tissue controls; the final values were estimated to be approximately $16 \%$ and $27 \%$ of age- and tissue-matched control, respectively. MtDNA copy numbers for the patient were still higher than that in reference DNA from blood ( $\sim 240 \%)$; however, as shown in Figure 3A, standard reference muscle and liver DNAs gave values of $\sim 1500 \%$ and $700 \%$ for muscle and liver, respectively when compared with a blood-derived reference. Thus, highly significant depletion could still be demonstrated using DNA from blood as the reference. DNA isolated from blood of the father and the carrier mother gave aCGH ratios within the normal range of $100 \%$ to $150 \%$ compared with reference DNA from blood.

Furthermore, from the same aCGH experiment, a deletion that included exons 1 and 2 of the TK2 gene was detected, which was inherited from the mother (Fig. 3B). Subsequent $\mathrm{PCR} /$ sequencing analysis revealed that the deletion encompassed $\sim 5.8 \mathrm{~kb}$ from nucleotide position $65,136,258$ to $65,142,085$ covered by 28 oligonucleotide probes.

\section{DISCUSSION}

Oligonucleotide aCGH has now been routinely used for the detection of large chromosomal deletions. ${ }^{4,5}$ On the basis of similar approaches, we have recently developed an oligonucleotide array targeted to a group of genes that are involved in metabolic and mitochondrial disorders. ${ }^{7}$ We have demonstrated that such an oligonucleotide array is useful when combined with direct DNA sequencing, particularly in autosomal recessive 

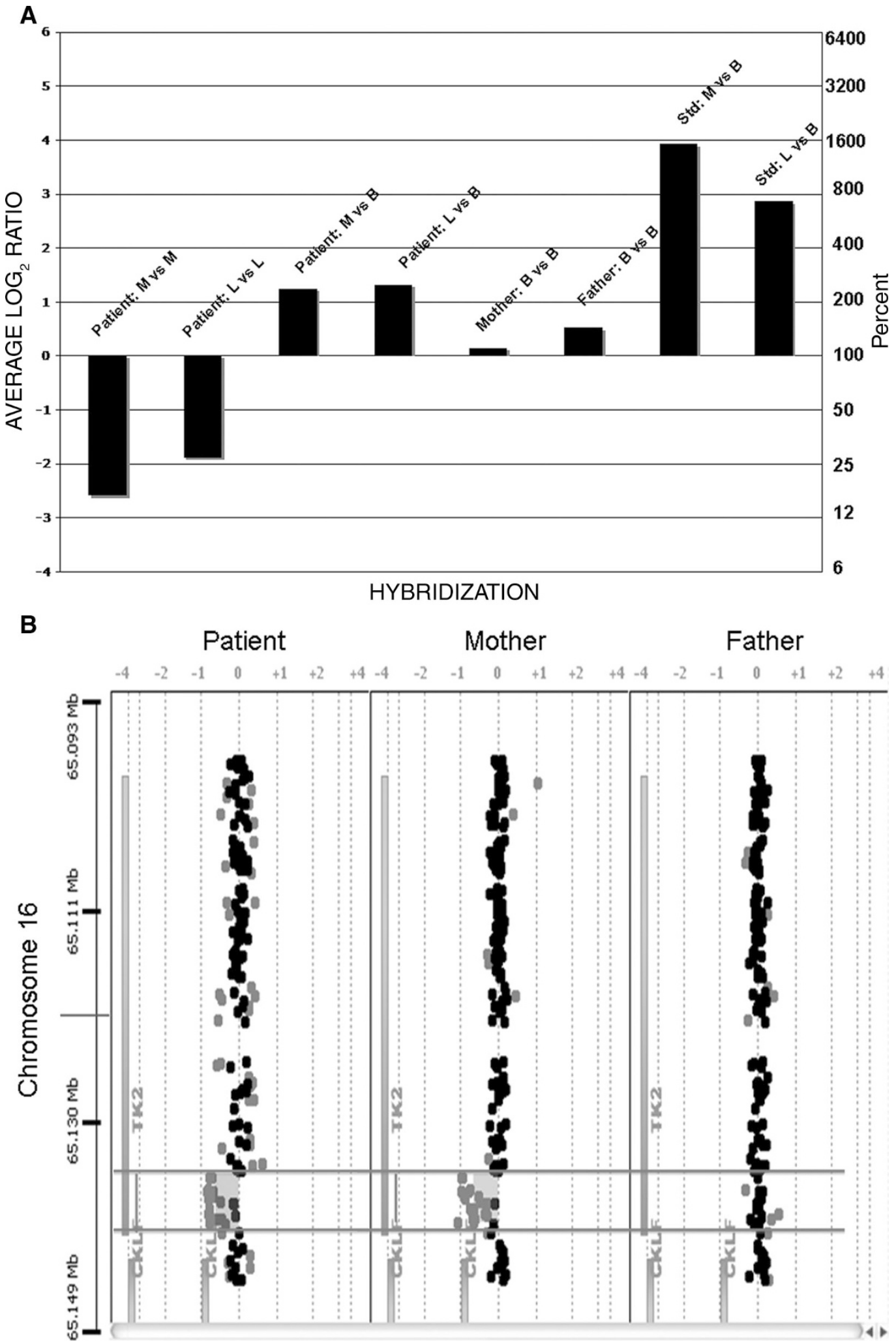

Fig. 3. Demonstration of mitochondrial DNA depletion associated with mutation of the TK2 gene. A, Shows the average $\log 2$ ratios for relative hybridization intensities of all mtDNA probes for the aCGH hybridizations indicated. The first four experiments were done with the patient DNA, the next two with parental DNAs, and the last two with standard laboratory samples. For patient and reference DNAs used, the sources are indicated as follows: $M=$ Muscle, $L=L i v e r$, and B $=B$ Bood. $B$, Analysis of the TK2 gene region showing a small deletion encompassing exons 1 and 2 in the patient and mother. 
diseases when only one heterozygous mutant allele is detected. In this report, we substantiate our application and expand it to include the mitochondrial genome deletion and depletion analysis.

To date, diagnosis of mtDNA deletion syndrome has relied on the demonstration of large mtDNA deletions by Southern analysis. As we have described earlier, the procedures are labor intensive and time consuming. Extensive experiments are required for restriction-fragment-length polymorphism mapping of the location of the deletion and determination of size of deletion and the percentage of deletion heteroplasmy.23,24 Utilization of the MitoMet array simplified the procedures to a single step of $\mathrm{CGH}$, which revealed not only the size and location of the deletion but also the percentage of deletion heteroplasmy. Because of the high-density coverage of the mitochondrial genome, most of the break points of deletions can be determined within 10 to $30 \mathrm{bp}$ precision by the oligonucleotide array (Table 1). Because the location and size of large mtDNA deletion do not correlate with disease clinical phenotype or severity, ${ }^{27}$ the break points elucidated by oligonucleotide aCGH are sufficient for diagnosis. Thus, tedious PCR/ sequencing procedures can be eliminated.

The percentage of deletion mutant heteroplasmy as determined by the oligonucleotide aCGH and densitometric scanning methods, in general, is within $20 \%$ agreement and typically very similar (Table 1). Some of the samples were analyzed with the MitoMet chip twice with highly reproducible results. The densitometric measurement is not an accurate quantitative method due to problems of high background or saturated signal intensity. However, the array method uses the average of thousands of data points. Therefore, the percentage of heteroplasmy calculated from the array data is theoretically more reliable than the densitometric method. Conversely, the estimation of mtDNA copy number by array may not be as accurate as real-time qPCR method. This is because there are hundreds (blood and skin) and thousands (liver and muscle) more copies of mitochondria DNA than nuclear DNA per cell. The hybridization signal intensity of the mitochondrial genome must be normalized to the individual's nuclear gene first before the copy number of the individual is compared with the matched control. Because the difference in mitochondrial and nuclear gene copy number is about 3 to $4 \log 2$ units, the measurement of copy number using array data may not be accurate due to the saturation of the mtDNA signal.

To date, at least 9 nuclear genes, TYMP, POLG, DGUOK, TK2, SUCLA2, SUCLG1, RRM2B, and TWINKLE, have been reported to cause mtDNA depletion. ${ }^{12-22}$ These diseases are autosomal recessive. In the cases where direct DNA sequencing identifies only one heterozygous point mutation, heterozygous deletion on the other allele is a reasonable possibility. Our MitoMet oligonucleotide array was designed for this purpose to detect intragenic deletions in targeted genes. The finding of the intragenic heterozygous deletion of two exons in $T K 2$ gene clearly demonstrates the power of this MitoMet oligonucleotide array in molecular diagnosis. Furthermore, simultaneous detection of the copy number changes in both nuclear and mitochondrial genomes makes the utility of this custom array of tremendous value in the diagnoses of mitochondrial depletion syndromes.

\section{ACKNOWLEDGMENTS}

This study was supported, in part, by a National Institutes of Health award 5R01 CA100023 (L.-J.C.W.).

\section{REFERENCES}

1. Bejjani BA, Shaffer LG. Application of array-based comparative genomic hybridization to clinical diagnostics. J Mol Diagn 2006;8:528-533.

2. Cheung SW, Shaw CA, Yu W, et al. Development and validation of a CGH microarray for clinical cytogenetic diagnosis. Genet Med 2005;7:422-432.

3. Lu X, Shaw CA, Patel A, et al. Clinical implementation of chromosomal microarray analysis: summary of 2513 postnatal cases. PLOS ONE 2007;2: e327.

4. Ou Z, Kang SH, Shaw CA, et al. Bacterial artificial chromosome-emulation oligonucleotide arrays for targeted clinical array-comparative genomic hybridization analyses. Genet Med 2008;10:278-289.

5. Shen Y, Irons M, Miller DT, et al. Development of a focused oligonucleotide-array comparative genomic hybridization chip for clinical diagnosis of genomic imbalance. Clin Chem 2007;53:2051-2059.

6. del Gaudio D, Yang Y, Boggs BA, et al. Molecular diagnosis of Duchenne/ Becker muscular dystrophy: enhanced detection of dystrophin gene rearrangements by oligonucleotide array comparative genomic hybridization. Hum Mutat 2008;29:1100-1107.

7. Wong LJ, Dimmock D, Geraghty MT, et al. Utility of oligonucleotide array-based comparative genomic hybridization for detection of target gene deletions. Clin Chem 2008;54:1141-1148.

8. DiMauro S, Davidzon G, Hirano M. A polymorphic polymerase. Brain 2006;129(Pt 7):1637-1639.

9. Ostergaard E, Christensen E, Kristensen E, et al. Deficiency of the alpha subunit of succinate-coenzyme A ligase causes fatal infantile lactic acidosis with mitochondrial DNA depletion. Am J Hum Genet 2007;81:383-387.

10. Ostergaard E, Hansen FJ, Sorensen N, et al. Mitochondrial encephalomyopathy with elevated methylmalonic acid is caused by SUCLA2 mutations. Brain 2007; 130(Pt 3):853-861.

11. Spinazzola A, Zeviani M. Disorders of nuclear-mitochondrial intergenomic communication. Biosci Rep 2007;27:39-51.

12. Bourdon A, Minai L, Serre V, Jais JP, et al. Mutation of RRM2B, encoding p53-controlled ribonucleotide reductase (p53R2), causes severe mitochondrial DNA depletion. Nat Genet 2007;39:776-780.

13. Carrozzo R, Dionisi-Vici C, Lucioli S, et al. SUCLA2 mutations are associated with mild methylmalonic aciduria, Leigh-like encephalomyopathy, dystonia and deafness. Brain 2007;130(Pt 3):862-874.

14. Dimmock DP, Zhang Q, Dionisi-Vici C, et al. Clinical and molecular features of mitochondrial DNA depletion due to mutations in deoxyguanosine kinase. Hum Mutat 2008;29:330-331.

15. Elpeleg O, Miller C, Hershkovitz E, et al. Deficiency of the ADP-forming succinyl-CoA synthase activity is associated with encephalomyopathy and mitochondrial DNA depletion. Am J Hum Genet 2005;76:1081-1086.

16. Galbiati S, Bordoni A, Papadimitriou D, et al. New mutations in TK2 gene associated with mitochondrial DNA depletion. Pediatr Neurol 2006;34:177185.

17. Hakonen AH, Isohanni P, Paetau A, Herva R, Suomalainen A, Lönnqvist T. Recessive Twinkle mutations in early onset encephalopathy with mtDNA depletion. Brain 2007;130(Pt 11):3032-3040.

18. Horvath R, Hudson G, Ferrari G, et al. Phenotypic spectrum associated with mutations of the mitochondrial polymerase gamma gene. Brain $2006 ; 129$ (Pt 7):1674-1684

19. Nishino I, Spinazzola A, Hirano M. Thymidine phosphorylase gene mutations in MNGIE, a human mitochondrial disorder. Science 1999;283:689-692.

20. Sarzi E, Goffart S, Serre V, et al. Twinkle helicase (PEO1) gene mutation causes mitochondrial DNA depletion. Ann Neurol 2007;62:579-587.

21. Wong LJ, Brunetti-Pierri N, Zhang Q, et al. Mutations in the MPV17 gene are responsible for rapidly progressive liver failure in infancy. Hepatology 2007;46:1218-1227.

22. Wong LJ, Naviaux RK, Brunetti-Pierri N, et al. Molecular and clinical genetics of mitochondrial diseases due to POLG mutations. Hum Mutat 2008;29:E150-E172

23. Lacbawan F, Tifft CJ, Luban NL, et al. Clinical heterogeneity in mitochondrial DNA deletion disorders: a diagnostic challenge of Pearson syndrome. Am J Med Genet 2000;95:266-268.

24. Wong LJ, Boles RG. Mitochondrial DNA analysis in clinical laboratory diagnostics. Clin Chim Acta 2005;354:1-20.

25. Bai RK, Wong LJ. Simultaneous detection and quantification of mitochondrial DNA deletion(s), depletion, and over-replication in patients with mitochondrial disease. J Mol Diagn 2005;7:613-622.

26. Olshen AB, Venkatraman ES, Lucito R, Wigler M. Circular binary segmentation for the analysis of array-based DNA copy number data. Biostatistics 2004;5:557-572

27. Wong LJ. Recognition of mitochondrial DNA deletion syndrome with nonneuromuscular multisystemic manifestation. Genet Med 2001;3:399-404. 